\title{
Experiencia de trabajo para la configuración del ERP Odoo en pequeños negocios. Caso de éxito en TostoneT
}

\author{
ERP Odoo configuration experience for small business. Successful case in TostoneT
}

Yanelis Pavón González ${ }^{1 *} \quad$ Liber Puente Baró ${ }^{2} \quad$ Marta Infante Abreu ${ }^{1} \quad$ Jeffrey Blanco González ${ }^{1}$

Recibido 15 de mayo de 2016, aceptado 1 de agosto de 2017

Received: May 15, 2016 Accepted: August 1, 2017

\section{RESUMEN}

En la actualidad, la información se ha convertido en un recurso estratégico de las organizaciones para desarrollar capacidades competitivas y de gestión. Esta afirmación también es válida para los pequeños negocios que deben prepararse para ser ágiles y certeros ante los cambios y oportunidades del entorno. Los sistemas de Planificación de Recursos Empresariales (ERP) contribuyen a fortalecer las capacidades de gestión de los negocios. Sin embargo, a pesar de sus ventajas estratégicas, el ciclo de aprendizaje para su configuración, es muy costoso. Este artículo expone la experiencia de trabajo de los autores para llevar a cabo un proyecto de aprendizaje y configuración del sistema ERP Odoo de manera intencionada, en el alcance de los procesos primarios de la cadena de valor. La aplicación se realizó en un pequeño negocio de informática, TostoneT, cuyos principales resultados fueron la reducción del ciclo de cierre contable, aprovechamiento de oportunidades de negocio y mayor conocimiento sobre administración de negocio.

Palabras clave: Cadena de valor, pequeños negocios, capacidades de los sistemas ERP, guía de trabajo.

\begin{abstract}
Today, information has become a strategic resource for organizations to develop competitive and management capabilities. This statement is also valid for small businesses to be prepared to be agile and accurate before changes and opportunities in the environment. Systems Enterprise Resource Planning $(E R P)$ contribute to strengthening the capacity of business management. However, despite its strategic advantages, the learning cycle for configuration is costly. This article presents the work experience of the authors to carry out intentionally a learning and configuration project of ERP system Odoo, within the scope of the primary processes of the value chain. The application was made in a small informatics business whose main results were: time optimization, leveraging business opportunities and greater knowledge of business administration.
\end{abstract}

Keywords: Value chain, small business, ERP systems capabilities, work guide.

\section{INTRODUCCIÓN}

Los cambios que se han producido en los últimos años sugieren el desarrollo de la era de la información y el conocimiento. Estos cambios se asocian fundamentalmente al acelerado desarrollo de las tecnologías de la información (TI); así como al consecuente crecimiento del volumen, capacidad y velocidad de intercambio de datos, información y conocimiento [1-3].

\footnotetext{
1 Instituto Superior Politécnico José Antonio Echeverría. Calle 114 No 11901. La Habana, Cuba.

E-mail: nelispavon@gmail.com, miabreu@ind.cujae.edu.cu, jblanco@ind.cujae.edu.cu

2 TostoneT: Servicios de Informática y Comunicación. Calle K, No 422, La Habana, Cuba. Email: libito@ gmail.com

* Autor de correspondencia.
} 
Las organizaciones deben prepararse para ser más flexibles y establecer estrategias con el objetivo de adaptarse al entorno variable de las organizaciones en el que desarrollan sus acciones. Ante ambientes tan poco estables, los miembros de la organización $y$, en particular, su alta gerencia necesita manipular grandes volúmenes de información para cumplir con sus actividades de gestión [4].

En este sentido, Cuba hoy proyecta políticas para concebir la informatización de los gobiernos, empresas y otras instituciones que garantice la inversión en TI acorde a los requerimientos de las organizaciones, con lo que preparan los presupuestos para invertir en estos proyectos que son bastantes costosos $[5,6]$.

Sin embargo, se le ha prestado poca atención a los pequeños negocios que han comenzado a desarrollarse de manera gradual e intentan sobrevivir día a día. Estos tipos de negocios se conciben con poca visión estratégica de los beneficios de las TI y las buenas prácticas de los procesos administrativos y de gestión.

Muchas empresas del mundo optan por realizar su informatización mediante los sistemas de Planificación de Recursos Empresariales (ERP, por sus siglas en inglés); ya que estos sistemas abarcan una amplia gama de aplicaciones software que soportan las operaciones de negocios diarias y la toma de decisiones basado en la integración de los dominios funcionales en las organizaciones $[4,7,8]$.

En Cuba existe poca experiencia documentada de empresas o pequeños negocios que operen con sistema ERP y, por otro lado, en la literatura donde abordan temáticas sobre los ERP, no existe una metodología de trabajo que guíe con intencionalidad los pasos de despliegue para cubrir el alcance de su cadena de valor.

Teniendo en cuenta los elementos anteriores, el siguiente trabajo tiene como objetivo: documentar la experiencia de trabajo de los autores, que guíe a los pequeños negocios cubanos a aprender con mayor rapidez los elementos que deben tener en cuenta en un proyecto de configuración del sistema Odoo en el alcance de su cadena de valor.

Para el cumplimiento de los objetivos, el trabajo se estructura de la siguiente manera:
- Las micros, pequeñas y medianas organizaciones en Cuba: Aborda el acelerado desarrollo de pequeños negocios en Cuba y sus problemáticas de gestión.

- Sistemas ERP para el desempeño de los pequeños negocios: Aborda las capacidades de los sistemas ERP para los pequeños negocios y problemáticas que resuelve.

- Odoo como opción para pequeños negocios: Caracteriza Odoo como una buena solución para pequeños negocios y las desventajas asociadas al aprendizaje.

- Experiencia de trabajo para la configuración de Odoo: Se propone una guía de trabajo basado en la experiencia de los autores.

- Configuración de Odoo en TostoneT: Se caracteriza el pequeño negocio y se muestran los resultados de impacto del sistema Odoo.

\section{LAS MICROS, PEQUEÑAS Y MEDIANAS ORGANIZACIONES EN CUBA}

Las micro pequeñas y medianas organizaciones $\left(\mathrm{MPyMO}^{3}\right)$ [10] tienen particular importancia para las economías nacionales, no solo por sus aportaciones a la producción y distribución de bienes y servicios, sino también por la flexibilidad de adaptarse a los cambios tecnológicos y gran potencial de generación de empleos. Representan un excelente medio para impulsar el desarrollo económico y una mejor distribución de la riqueza [11]. Sin embargo, presentan algunas limitaciones como:

- Dueños de negocio con poca formación o experiencia en la administración de negocio.

- Acceso limitado a las fuentes de financiamiento.

- Bajos niveles de innovación y desarrollo tecnológico.

- Poca penetración en mercados internacionales.

- Insuficientes niveles de productividad.

- Bajos niveles de asociación y colaboración.

No obstante, a pesar de sus limitaciones, el papel de las MPyMO en la economía del país ha sido un factor

\footnotetext{
3 Término equivalente utilizado en Cuba para referirse a las PYMES.

Mediana: cuando el número de trabajadores oscile entre 20 y 50. Pequeña: cuando el número de trabajadores oscile entre 11 y 19. Micro: cuando el número de trabajadores es menor de 10 [9] NC 1030: 2014. Modelo de gestión para mico, pequeña y medianas organizaciones, O.N. d. Normalización, 2014.
} 
que ha generado incrementos en la producción; valor agregado; aportaciones fiscales; fortalecimiento del mercado interno; aumento de exportaciones, etc., por lo que han sido motivo de diseño de políticas encaminadas a promoverlas y apoyarlas para elevar su competitividad y enfrentar la competencia de un mundo globalizado, sin olvidar el mundo de los emprendedores, donde las empresas líderes son aquellas que adoptan modelos de subcontratación y alianzas estratégicas con MPyMO, que gracias a su capacidad de adaptación y flexibilidad crecen en un mundo en constante cambio, demostrando además que cuando se organizan pueden superar las aparentes limitaciones de su tamaño [12].

Considerando la importancia de este modelo de gestión no estatal para las economías nacionales, Cuba ha iniciado, desde octubre de 2010, el nuevo proceso de ampliación del trabajo por cuenta propia, el que ha sido favorecido por medidas adoptadas en los últimos años para continuar su perfeccionamiento y flexibilización, el número de trabajadores inscritos en esta modalidad no estatal de gestión ha aumentado considerablemente (ver Figura 1) [13].

El trabajo por cuenta propia puede clasificar como microorganización, pero muchas veces, a pesar de su reducida cantidad de miembros, tienen altos niveles de gestión administrativa.

Por otra parte, también la formación de cooperativas no agropecuarias, ha tenido un crecimiento sostenido en el tiempo como se muestra en la Figura 2.

A pesar de las conocidas ventajas que tiene la creación de las cooperativas para el país, en el estudio que realiza Piñeiro [14] y noticias del

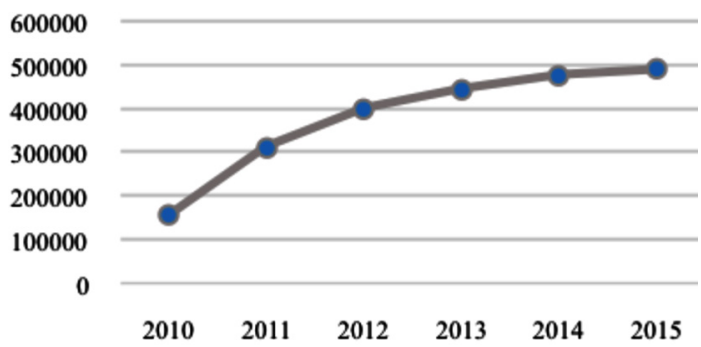

Figura 1. Número de personas que ejecutan el trabajo por cuenta propia.

Fuente: Elaborado por los autores basado en el análisis de los periódicos: Granma, Juventud Rebelde y Trabajadores.

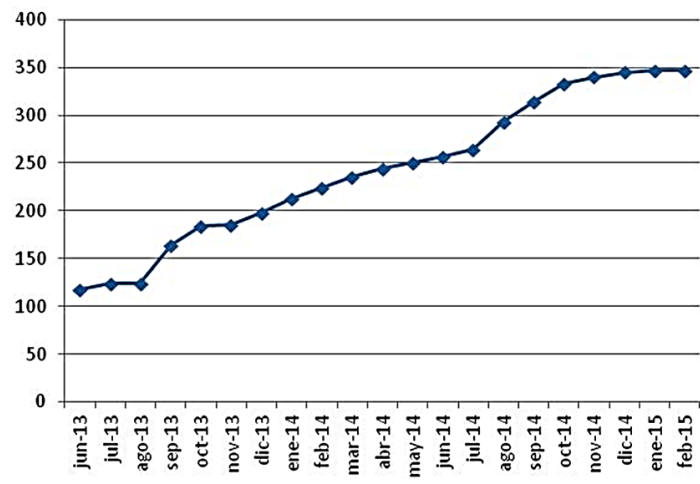

Figura 2. Cooperativas no agropecuarias constituidas. Fuente: [14]

periódico Trabajadores [15] se encuentra que el control interno tiene serias limitaciones.

- Proceso de creación con carácter experimental, cuya decisión es de forma inducida, promovida por entes externos al grupo de fundadores, limitando propiciar que sea una decisión de emprendimiento, consientes y voluntaria, lo que tributa a tener sentido de pertenencia.

- Insuficiencias en el control de gestión, ya que no existe la cultura de crear evidencias de las compra y ventas, lo que no permite mantener el control de los ingresos y gastos, limitando la fiabilidad de los análisis económicos.

- La mayoría de las cooperativas no se utilizan comprobantes de ventas y se realizan compras sin que quede constancia de ello.

- Desconfianza entre la administración de la cooperativa y el resto de los socios, pues eso atenta contra la cohesión interna tan necesaria para que la toma de decisiones democrática ocurra de forma efectiva.

- No tienen habilitado un registro consecutivo de las facturas; no existe un control de las facturas canceladas; no cuentan con modelos foliados por áreas contables.

- Inexistencia de evidencia del balance de apertura de las operaciones (estipulado en la Resolución $\mathrm{N}^{\circ} 427 / 012$ del MFP) [16] y que los balances de comprobación de saldos no tienen suficiente legitimidad; entre otras dificultades.

En este punto no se debe dejar de tener en cuenta que la creación y gestión de las MPyMEs también debe ser con carácter estratégico que garantice la 
sostenibilidad en el tiempo, por lo que, desde sus inicios, se deben contemplar las buenas prácticas de gestión ya que el mundo existen experiencias exitosas que implementan este modelo de gestión [17].

La sintomatología que se evidencia en la descripción de las problemáticas anteriores, son el resultado de una mala gestión de la información, siendo este un recurso fundamental en la actual sociedad digital. En este sentido, el enfoque de servicio al cliente va ganando fuerza, lo cual provoca que las empresas evalúen sus procesos internos y regeneren los conceptos de su cadena de valor a través de la integración y la automatización [18]. Esto es importante no solo para resolver los problemas de gestión de la información con los que se enfrentan las MPyMO cubanas sino para estar preparado para competir ante las nuevas oportunidades de negocio en Cuba.

\section{SISTEMAS ERP PARA EL DESEMPEÑO DE LOS PEQUEÑOS NEGOCIOS}

Actualmente, las empresas tienen una visión de negocio distinta a la de hace años, y es que, si una empresa quiere generar más ingresos, deben tener un control exhaustivo y prácticamente a tiempo real de todo lo que está pasando en su negocio, para gestionar con solvencia todos los recursos, minimizando tanto como sea posible los costos, pudiendo así sacar el máximo rendimiento otorgando grandes beneficios [19].

Por esta razón, surgen los sistemas ERP, substituyendo a los antiguos y obsoletos programas de gestión. Es un sistema de información que integra y maneja gran parte del negocio de muchas empresas, tratando con un gran volumen de información. Éstos se suelen dividir en módulos, facilitando así su instalación y configuración, adaptándose en todo momento a las necesidades y características de la empresa dependiendo de a qué se dedique la misma; ya que no es lo mismo una empresa que se dedica a la producción que una que se dedica al sector servicios [18].

Con los ERP, las transacciones quedan registradas desde su captura en una sola base de datos, en la cual se puede consultar en línea la información relevante. Las ventajas que ofrece la implementación de un sistema ERP son: Control de la operación, eficiencia administrativa, productividad, servicio a clientes, ahorros en costos operativos, visibilidad de las operaciones, soporte a toma de decisiones, entre otras; apoyando a distintas áreas de la organización como: compras, recursos humanos, almacenes, finanzas y mercadotecnia [20].

Cada empresa es un conjunto de actividades que se desempeñan para diseñar, producir, llevar al mercado, entregar y apoyar a sus productos. Todas esas actividades pueden ser representadas usando una cadena de valor [21] como se puede ver en la Figura 3.

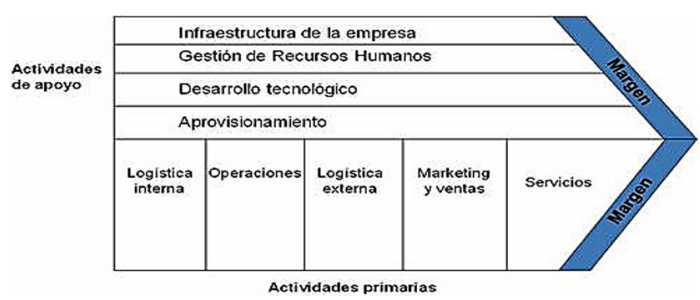

Figura 3. Cadena de valor de Porter. Fuente: [21].

Logística interna: La primera actividad primaria de la cadena de valor es la logística interna. Las empresas necesitan gestionar y administrar una manera de recibir y almacenar las materias primas necesarias para crear su producto, así como el medio para distribuir los materiales. Cuanto más eficiente sea la logística interna, mayor es el valor generado en la primera actividad.

Operaciones: La siguiente etapa de la cadena de valor son las operaciones. Las operaciones toman las materias primas desde la logística de entrada y crea el producto. Naturalmente, mientras más eficientes sean las operaciones de una empresa, más dinero la empresa podrá ahorrar, proporcionando un valor agregado en el resultado final.

Logística Externa: Después de que el producto está terminado, la siguiente actividad de la cadena de valor es la logística de salida. Aquí es donde el producto sale del centro de la producción y se entrega a los mayoristas, distribuidores, o incluso a los consumidores finales dependiendo de la empresa.

Marketing y Ventas: Marketing y ventas es la cuarta actividad primaria de la cadena de valor. Aquí hay 
que tener cuidado con los gastos de publicidad, los cuales son una parte fundamental de las ventas.

Servicios: La actividad final de la cadena de valor es el servicio. Los servicios cubren muchas áreas, que van desde la administración de cualquier instalación hasta el servicio al cliente después de la venta del producto. Tener una fuerte componente de servicio en la cadena de suministro proporciona a los clientes el apoyo y confianza necesaria, lo que aumenta el valor del producto.

Los negocios que incorporen los sistemas ERP heredan las buenas prácticas de los procesos que automatiza, con lo que, se incrementaría la curva de aprendizaje del negocio para asimilar el conocimiento de los procesos de gestión; contribuyendo, principalmente, a desarrollar capacidades en la cadena de valor del negocio [22].

\section{ODOO COMO OPCIÓN PARA PEQUEÑOS NEGOCIOS}

Los sistemas ERP mejor rankeados en el mundo son propietarios y muy costosos sin embargo, se han desarrollado varios sistemas ERP libres de costo de adquisición, el que se convierte en una solución viable para pequeños negocios con pocas capacidades de financiamiento [23].

Entre los sistemas ERP libres de costo de adquisición en el mercado, se ha despertado un gran interés por el sistema Odoo 8 desde el año 2014 (ver Figura 4), también conocido como OpenERP 8.

Odoo usa el paradigma cliente-servidor (ver Figura 5). El cliente corre en una aplicación Javascript en el navegador, conectado al servidor usando el protocolo

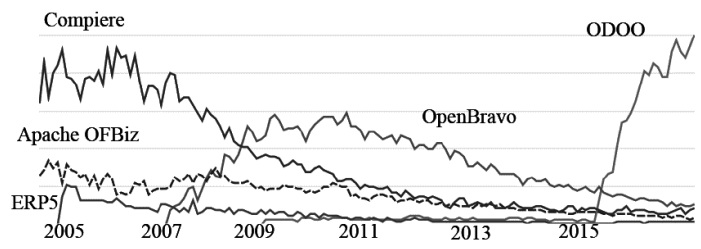

Figura 4. Interés de búsqueda sobre Sistemas ERP libres.

Fuente: Herramienta de Google Trends www.google. com/trends/explore (Consultado 3-02-2016).
JSON-RPC sobre HTTP(S). Al momento los clientes pueden conectarse al servidor usando XML-RPC o JSON-RPC.

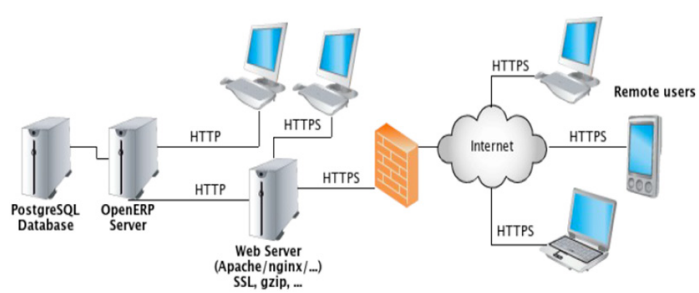

Figura 5. Arquitectura Odoo.

Fuente: Especificaciones técnicas de Odoo.

Actualmente cuenta con la presencia en más de 150 países con una gran comunidad detrás ayudando a su desarrollo y corrección de errores. La aplicación cuenta con 2 millones de usuarios en el mundo y tan solo en España tiene 150.000 usuarios, siendo el ERP favorito de las pequeñas y medianas empresas [24]. Cuenta con más de 1.800 módulos de los cuales aproximadamente 200 son oficiales [24]. La Figura 6 muestra los módulos de base del sistema.

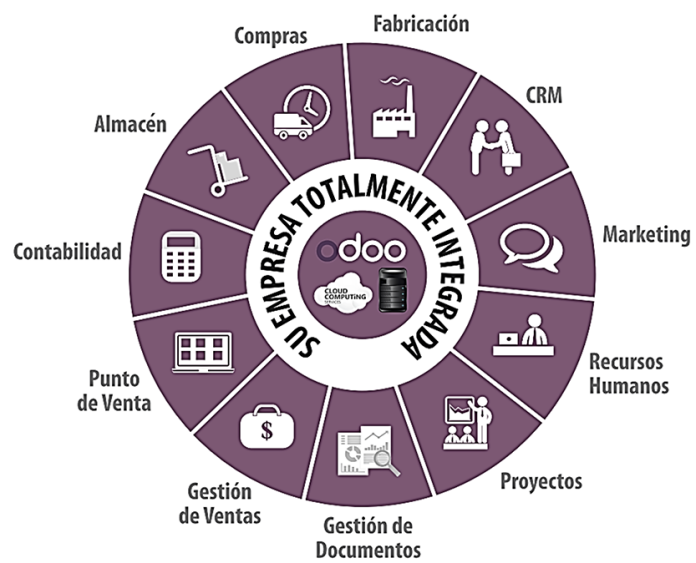

Figura 6. Módulos de base de Odoo.

Fuente: http://grupoadap.com (Consultado 3-022016).

La caracterización de Odoo en la literatura evidencia muchas ventajas beneficiosas para los pequeños negocios de acuerdo a las tendencias de los sistema ERPs descritas en el acápite anterior [25, 26]; sin embargo, su introducción en las organizaciones requiere un esfuerzo de aprendizaje por parte de 
los analistas organizacionales para configurarlo y adaptarlo a los procesos de negocio.

Para ello existen manuales, tutoriales y metodología de instalación y configuración del sistema, tal y como se muestra en la Tabla 1; pero no existe un método de trabajo que guíe con intencionalidad, la configuración de Odoo en base a la cadena de valor de las pequeñas organizaciones, con lo que se pueda priorizar y concentrar esfuerzos de aprendizaje en los temas de interés (dimensión pragmática), de manera tal que garantice la escalabilidad del aprendizaje.

Goeun Seo [19] reconoce diferentes factores que provocan el fracaso de proyectos ERP, entre los que se pueden mencionar: la falta de una metodología de gestión de proyecto eficaz, insuficiencias en la conformación del equipo de trabajo, poca focalización en los alcances definidos y problemas en el rediseño de los procesos. Basado en la experiencia de los autores, han ocurrido fracasos, como consecuencias de dichos factores que a continuación se mencionan:

Tabla 1. Estrategia de búsqueda asociada a la existencia de manuales, tutoriales y metodologías de configuración de Odoo.

\begin{tabular}{|c|c|c|c|}
\hline Frase de búsqueda & Ref. & Frase de búsqueda & Ref. \\
\hline manual erp Odoo & 67.000 & $\begin{array}{l}\text { "pyme" "Odoo" } \\
\text { "erp" filetype:pdf }\end{array}$ & 67 \\
\hline method erp Odoo & 51.800 & $\begin{array}{l}\text { "cadena de valor" } \\
\text { "Odoo" "erp" } \\
\text { filetype:pdf }\end{array}$ & 45 \\
\hline $\begin{array}{l}\text { methodology erp } \\
\text { Odoo }\end{array}$ & 25.300 & $\begin{array}{l}\text { "cadena de valor" } \\
\text { "Odoo" "erp" metodo } \\
\text { filetype:pdf }\end{array}$ & 8 \\
\hline metodo erp Odoo & 16.000 & chain value Odoo erp & 6 \\
\hline $\begin{array}{l}\text { "pequeño negocio" } \\
\text { "Odoo" "erp" }\end{array}$ & 9.640 & $\begin{array}{l}\text { "pequeño negocio" } \\
\text { "Odoo" "erp" } \\
\text { filetype:pdf }\end{array}$ & 2 \\
\hline $\begin{array}{l}\text { metodología erp } \\
\text { Odoo }\end{array}$ & 9.090 & $\begin{array}{l}\text { "metodo" "pyme" } \\
\text { "Odoo" "erp" } \\
\text { filetype:pdf }\end{array}$ & 2 \\
\hline $\begin{array}{l}\text { "supply chain" } \\
\text { "Odoo" "erp" } \\
\text { filetype:pdf }\end{array}$ & 370 & $\begin{array}{l}\text { "short business" } \\
\text { "Odoo" "erp" } \\
\text { filetype:pdf } \\
\end{array}$ & 1 \\
\hline $\begin{array}{l}\text { "methodology" } \\
\text { "Odoo" "erp" } \\
\text { filetype:pdf }\end{array}$ & 199 & & \\
\hline
\end{tabular}

Fuente: Elaboración de los autores.
- Interés por conocer los módulos con profundidad sin tener en cuenta la dimensión pragmática de lo que deseaban informatizar, lo cual atentaba con la agilidad del proyecto.

- Selección aleatoria de las necesidades de conocimiento en el consumo de los manuales y tutoriales sin el orden adecuado, lo que provocaba en muchos casos, comenzar desde el principio.

- Tendencia a iniciar rápidamente la configuración del ODOO, sin un trabajo de mesa para estructurar la información del diseño en colaboración con los especialistas del negocio, lo que implicaba brechas entre lo que se configuraba y las especificaciones del negocio. En este caso también había que comenzar desde el principio.

Habiendo experimentado estas problemáticas, se propone una guía de trabajo para aprender y configurar Odoo, la cual permite identificar las necesidades de conocimiento e ir directo al acápite de los manuales y tutoriales que se necesiten.

\section{EXPERIENCIA DE TRABAJO PARA LA CONFIGURACIÓN DE ODOO}

La siguiente guía de trabajo es válida para la configuración del sistema Odoo 8 en el alcance de las capacidades de negocio que se representan en la Figura 7. La guía solo está definida para informatizar los procesos primarios definidos en la cadena de valor de Porter, aunque el sistema

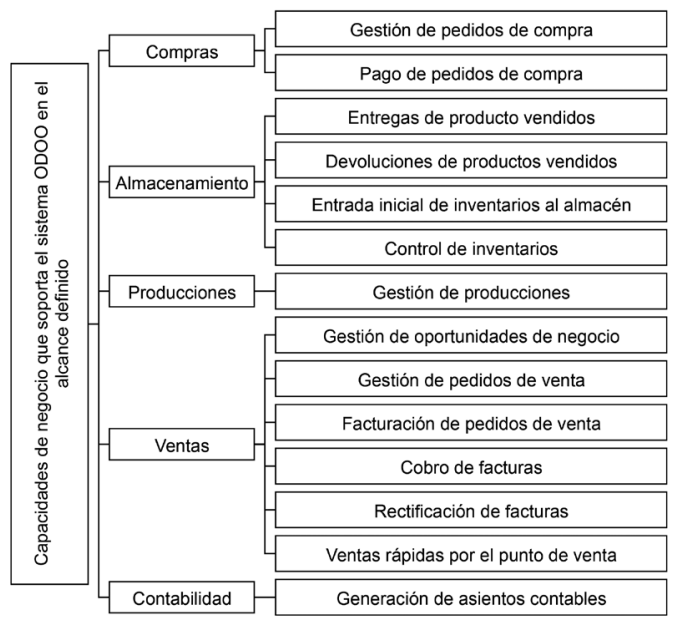

Figura 7. Capacidades de negocio que soporta el sistema.

Fuente: Elaboración propia. 
puede cubrir otros procesos no definidos en esta propuesta. Se incorpora la contabilidad ya que todas las operaciones tienen una implicación contable.

Para llevar a cabo un proyecto de configuración de cualquier sistema ERP es necesario cumplir con los siguientes presupuestos.

- Compromiso de la alta dirección para propiciar el cambio.

- Manejo de un vocabulario común en base a la conceptualización de ERP.

- Disposición para incorporar la buena práctica administrativa que concibe el sistema en los procesos de la organización.

- Conocimiento sobre los principales conceptos que maneja el negocio.

Los actores involucrados en un proyecto de configuración del sistema ERP son:

- Analista de sistema: Estudia y analiza el negocio y en consecuencia configura el sistema para satisfacer sus necesidades.

- Experto del negocio: Conocedores del modelo de negocio, información que maneja y sus procesos. Proveen las informaciones actuales del negocio que permita poblar las bases de datos.

- Expertos funcionales: Conocedores de los dominios de negocios que están siendo afectado por la configuración del sistema. Proveen conocimiento de las áreas funcionales.

- Trabajador del negocio: Usuarios del sistema que reciben capacitaciones para su uso. Registran las informacionales iniciales al sistema.

A continuación, se definen los pasos para el levantamiento de información del negocio y configuración de Odoo en el alcance descrito. Estos pasos son definidos a partir de la experiencia de trabajo de los autores. La Figura 8 ilustra la secuencia definida.

\section{Instalar módulos definidos en el alcance}

En el alcance del proyecto es requerido instalar los módulos que se muestran en la Figura 6.

CRM: Empuja la productividad de las ventas. Gestiona las iniciativas de negocio y organiza sus oportunidades. Seguimiento a llamadas telefónicas y reuniones.

Gestión de ventas: Maneja el funcionamiento de las ventas, juntamente con sus facturas, facturación totalmente integrada y guarda la huella de los contratos con clientes.

Contabilidad y finanzas: Gestiona toda la contabilidad. Puede acceder a el mapa de cuenta, asientos y apuntes contables, así como la generación de informes de contabilidad y finanzas. Gestiona cobros y pagos.

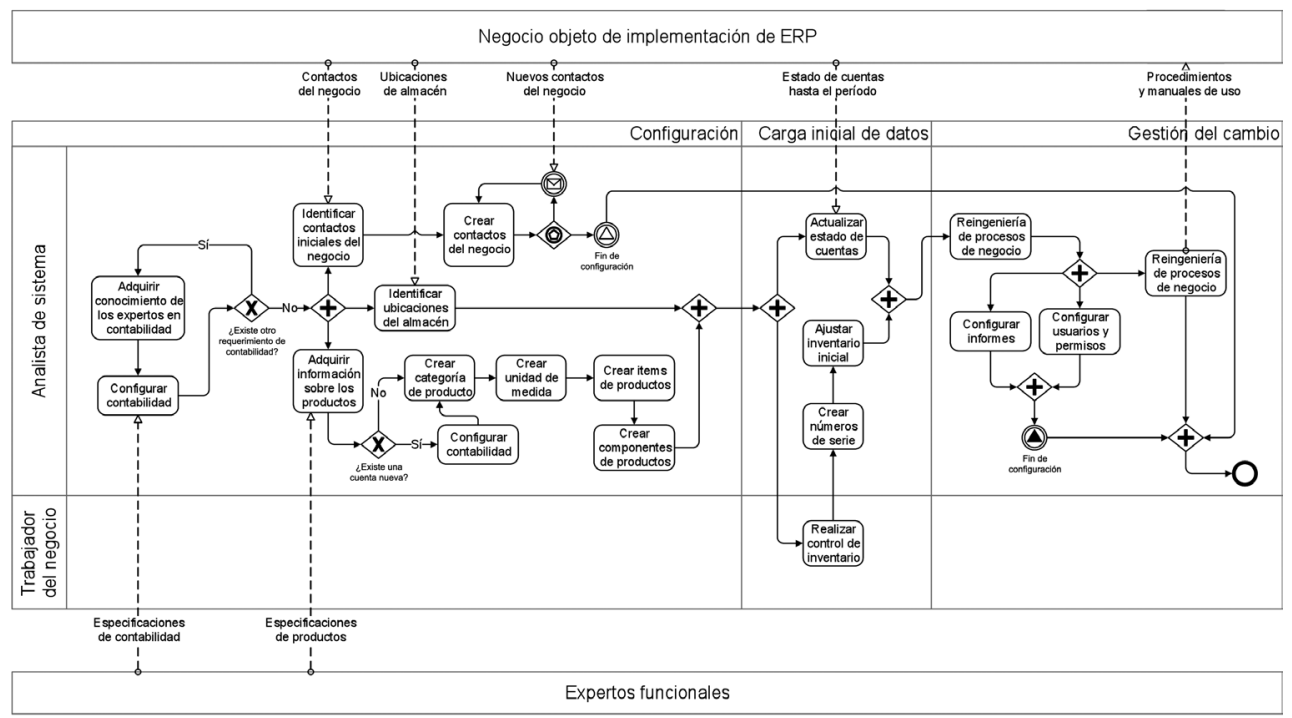

Figura 8. Experiencia de trabajo para configurar Odoo.

Fuente: Elaboración propia. 


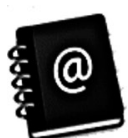

CRM

Iniciativas, oportunidades,

llamadas telefónicas

crm

Installed

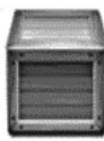

Gestión de almacenes Inventario, logística, almacenamiento stock

Installed

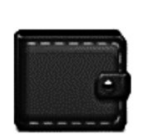

Contabilidad y finanzas

Contabilidad financiera y analítica account_accountant

Installed

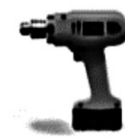

MRP (Planificación de Requerimientos de Materiales) Órdenes de fabricación, lista de materiales, rutas de producción mrp

Installed

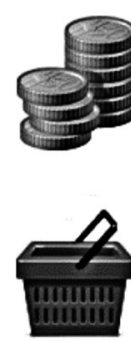

Gestión de ventas

Presupuesto, pedidos de venta, facturación

sale

Installed

Gestión de compras

Pedidos de compra, recibos y

facturas de proveedor

purchase

Installed

Figura 9. Módulos de Odoo instalados.

Fuente. Sistema Odoo.

Gestión de almacenes: Sistema de inventario de entrada doble. Automatiza las transacciones.

MRP: Planifica los recursos de materiales y maneja orden de producción.

Gestión de compras: Automatiza las compras desde el presupuesto hasta la factura.

\section{Adquirir conocimiento de los expertos de contabilidad}

En este paso es importante colaborar con expertos funcionales de contabilidad y finanzas y expertos del negocio que permita adquirir conocimiento sobre todas las operaciones transaccionales que tenga implicación en la contabilidad. Cada una de estas operaciones da lugar a un asiento contable de partida doble formado por las cuentas que maneja la empresa.

En Cuba, para los pequeños negocios existe un nomenclador de cuenta, así como para las empresas; que puede ser consultado [27] y que se encuentra estructurado en una jerarquía de cuentas; sin embargo, puede que el negocio no utilice todas las cuentas por su naturaleza. En este sentido, se identificaron todas las operaciones transaccionales del negocio a las que se le asociaron las cuentas que moviliza, dejando fuera del nomenclador, aquellas cuentas que el negocio no necesita utilizar.

Para la captura del conocimiento sobre las operaciones transaccionales de la empresa y el asiento asociado, se utilizó la planilla que se muestra en la Tabla 2, la cual contiene un ejemplo que ilustra la estructura informacional: con la que se identifica el sistema de cuenta pertinente al negocio.

\section{Configurar contabilidad}

Luego de identificar el nomenclador de cuenta se puede iniciar la configuración de la contabilidad en el sistema. Para agilizar el proceso de configuración puede modificar el sistema de cuenta en lugar de crear uno nuevo ya que es muy similar al cubano.

En el sistema aparecen por defecto un sistema de cuenta que puede modificar lo que posibilita ser más productivo en la configuración, ya que tiene una estructura muy similar a la cubana.

Tabla 2. Plantilla de asientos contables.

\begin{tabular}{|c|c|c|c|c|c|c|c|}
\hline Operación: & OP-01: Venta de un componente que aún no ha sido cobrado ni entregado \\
\hline Cód. Cta. & Cuenta & Cód. Subcta. & Subcuenta & Cód. Anál. & Análisis & Debe & Haber \\
\hline 900 & Venta de productos & 900,01 & Venta de componentes & - & - & & Aumenta \\
\hline 135 & Cuenta por cobrar & - & - & - & - & Aumenta & \\
\hline Operación: & OP-03: Entrega de productos asociado a un pedido & \multicolumn{5}{|l|}{} \\
\hline Cód. Cta. & Cuenta & Cód. Subcta. & Subcuenta & Cód. Anál. & Análisis & Debe & Haber \\
\hline 189 & Mercancía para la venta & 189,01 & Inventario de componentes & - & - & Disminuye & \\
\hline 810 & Costo de venta & 810,01 & Costo de componentes & - & - & Aumenta & \\
\hline
\end{tabular}

Fuente: Tomado del negocio. 
Es muy importante chequear la existencia de los siguientes tipos de cuenta:

- Liquidez: Cuenta que opera con la entrada y salida de dinero al negocio.

- A cobrar: Cuenta con la que opera las ventas y cobros del negocio.

A pagar: Cuenta con la cual opera las compras, gastos, impuestos por pagar, así como los pagos del negocio.

Por otra parte, debe configurar en contabilidad los libros diarios. El Libro Diario o Libro de cuentas es un libro contable donde se recogen, día a día, los hechos económicos de una empresa. La anotación de un hecho económico en el Libro Diario se llama asiento; es decir en él se registran todas las transacciones realizadas por una empresa.

En el sistema aparece ya configurado el diario de efectivo y el diario de banco, quienes se convierten en una forma de pago en las operaciones de cobro y pago. También están configurado el diario de ventas donde quedan los hechos económicos asociado a las ventas; el diario de compras donde quedan recogidas las compras del negocio, el diario de inventarios donde se recogen los hechos asociado a los movimientos de inventarios que pueden ser del tipo: entregas, recepciones y pérdidas.

\section{Identificar contactos iniciales del negocio}

En el estado actual del negocio, si no es de nueva creación, ya debiera existir una base de contactos que se puede registrar en el sistema para agilizar la entrada de datos iniciales. En este caso se debe interactuar con los expertos del negocio para identificar la información que desea gestionar de cada uno de sus contactos de manera tal que garantice un adecuado conocimiento sobre ellos que le permita operar de manera táctica y estratégica. Un contacto puede ser un cliente, proveedor, empleados o socios.

\section{Creación de contactos}

El Odoo le permite registrar información de contacto de los socios del negocio la que puede ser compartida por todos los usuarios del sistema. También se configura la información asociada a la contabilidad que le permite operar con dicho socio cuando establece una relación de compra-venta; en este caso se le asocia la cuenta "A cobrar" o "A pagar". De manera general o particular puede crear cuestionarios en los perfiles de cada contacto para guardar información con la que puede realizar posteriormente procesamientos y análisis.

\section{Adquirir información sobre los productos}

Para mantener una buena comunicación entre los expertos del negocio y los expertos funcionales los analistas de sistema deben realizar las preguntas adecuadas que les permita adquirir la información requerida para la configuración del sistema. De las informaciones adquiridas los analistas deben ser capaces de identificar grupos de productos, variantes de productos, modo de almacenamiento, su función en el negocio, etc.

\section{Crear categoría de producto}

Luego de adquirir información de los productos puede crear agrupaciones teniendo en cuenta aquellos que tienen el mismo sistema de cuentas para operar, con los que crea las categorías de producto.

En Odoo, las categorías de producto le permiten configurar las cuentas asociadas a los productos similares una sola vez. Los productos que clasifiquen en una misma categoría tendrán la misma configuración de cuentas. La Figura 10 muestra un ejemplo.

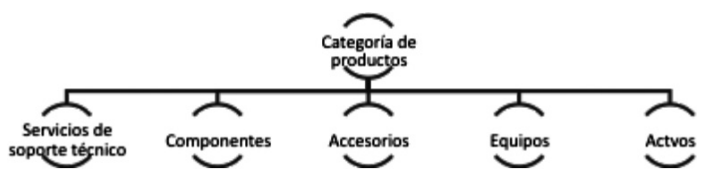

Figura 10: Ejemplo de categorías de productos. Fuente: Elaboración propia.

\section{Crear de unidades de medida}

La creación de unidades de medida le facilitará operar con los inventarios en el sistema, pues muchas veces se utilizan unidades de medidas diferentes en función del tipo de operación. Por ejemplo; usted puede comprar 1T de azúcar y solo utilizar en la producción de una taza de café $1 \mathrm{mg}$, en este caso se hace muy arduo operar rápidamente con esta diferencia. La configuración de las unidades de medida le facilita el trabajo.

\section{Crear de ítemes de productos}

Luego puede crear los ítemes de productos que clasifican en una categoría, adicionándole la 
información que adquirió en el paso 6. Es importante que tenga en cuenta que:

- Aunque defina un precio de costo y un precio de venta, este puede ser modificado en el momento que se realiza la compra o la venta.

- En método de costo se especifica precio estándar para los productos cuyo valor de inventario siempre serán el mismo, independientemente del precio de adquisición. Se especifica precio medio para productos cuyo valor de inventarios será la media de los precios de adquisición de todos los productos que han sido comprados. Se especifica precio real para los productos cuyo valor de inventario será exactamente igual al precio de adquisición del producto.

- En variante se crean productos hijos del que se está editando a partir de características específicas, por ejemplo: usted está creando el producto Memorias RAM y desea especificar la tecnología y la capacidad como atributos para este producto cuyos valores serían: DDR, DDR2 o DDR3 y $1 \mathrm{~Gb}, 2 \mathrm{~Gb}$ o $3 \mathrm{~Gb}$, respectivamente. En este caso se crearían tantas variantes como combinaciones de valores de atributos haya.

\section{Crear composiciones de los productos}

Existen productos que se fabrican a partir de materias primas o servicios que consumen productos, para ello se debe especificar la lista de materiales y la norma de consumo requerido para ello. Este paso es importante ya que garantiza que el sistema gestione adecuadamente los inventarios que constituyen componente del producto que se está vendiendo. Los tipos de productos que tendrán asociado una lista de materiales será aquellos que hayan clasificados como:

- Servicios que se venden y consumen materias primas en el momento de ser vendidos.

- Productos que se producen para ser almacenados y luego venderse o consumirse.

\section{Actualizar estados de cuenta}

Si el negocio no es de nueva creación debe identificar el saldo de cada una de las cuentas, ya que el sistema por defecto las tiene en cero. De esta manera garantiza la continuidad de las transacciones contables.

\section{Realizar control de inventario}

Para el control de los inventarios, es necesario hacer una actualización y levantamiento del inventario físico para luego ponerlo en el sistema, siendo necesario definir de estos, el nombre del producto, el número de lote o el número de serie, que permitirá posteriormente garantizar la trazabilidad en el sistema. El número de serie es un número alfanumérico único asignado para la identificación de lotes. Se utiliza comúnmente para identificar un objeto en particular dentro de una gran cantidad de estos. Para el levantamiento de la información sobre los inventarios, puede seguirse la estructura secuencial de pasos que por defecto propone Odoo. De esta forma cuando se solicite el inventario físico por el propio sistema, este puede ser ajustable fácilmente.

\section{Crear números de serie}

Una vez identificado los números de lote o número de serie, se puede comenzar a crearlos en el sistema. Ello le garantizará seguir la trazabilidad de los productos el sistema; por ejemplo: cuando usted vende una unidad de producto el sistema generará el asiento asociado a la salida de producto del inventario con su contrapartida de costo de venta, cuyo valor está asociado al precio de adquisición del producto; con lo que usted podrá calcular la rentabilidad de la venta de ese producto. Además, ante cualquier incidencia con el producto usted podrá consultar la vía de adquisición, entre otras operaciones que le garantiza el conocimiento de las operaciones de su negocio.

\section{Ajustar inventario inicial}

Finalmente realiza el ajuste de inventarios, actualizando las cantidades actuales por cada lote de producto. Con ello garantiza que el sistema pueda comenzar a operar con los productos en existencia.

\section{Reingeniería de procesos de negocio}

Redefinir los procesos de la organización acorde al flujo de trabajo de Odoo, de manera tal que se ajuste a la buena práctica embebida en el sistema. Explicitar los procedimientos e instrucciones de trabajo. Los resultados de este paso contribuyen a la transferencia efectiva del conocimiento, lo que garantiza la eficiencia del aprendizaje de los usuarios del sistema y las colaboraciones en la ejecución de los procesos.

\section{Configuración de informes}

Definir los indicadores relevantes que debe manejar el negocio para el análisis y toma de decisiones a partir de los datos de las compras, ventas, productividad del trabajo, análisis contable, etc. 


\section{Configuración de usuarios y permisos}

A partir de la definición de los procesos se podrán identificar las funciones específicas de cada usuario para la configuración de los permisos de acceso.

\section{Transferencia de conocimiento}

Se inicia el entrenamiento y acompañamiento para el uso del sistema que garantice una explotación estable.

\section{CONFIGURACIÓN DE ODOO EN TOSTONET}

Tostone T es una cooperativa en formación que aúna a emprendedores cubanos que brindan soluciones informáticas y de comunicación basada en la gestión del conocimiento, priorizando el uso de tecnologías sustentables y libres para impactar clientes en el mercado nacional e internacional.

Para ello cuenta con un taller de soporte técnico a equipos de cómputo, que brinda soluciones a su comunidad, personas que llegan al taller y a empresas estatales.

Cada día sus directivos se preocupan por elevar su eficiencia organizacional, participando en cursos de superación para emprendedores y consumiendo bibliografía sobre la gestión de pequeños negocios. Sin embargo, han identificados problemáticas que todavía limitan sus capacidades de gestión, tales como:

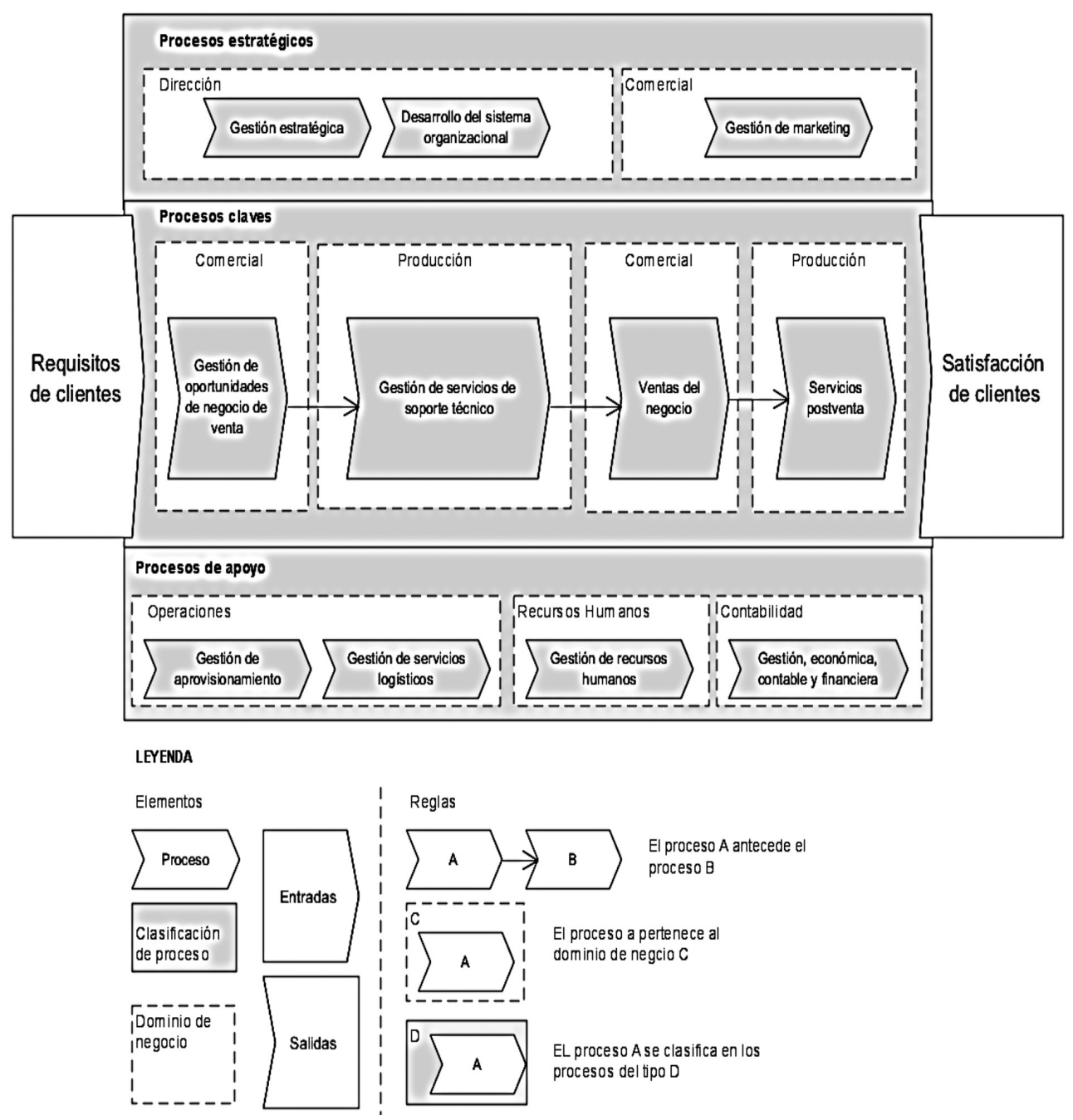

Figura 11: Mapa de proceso TostoneT.

Fuente: Tomado del negocio. 
- No existe claridad de las piezas disponibles para las reposiciones.

- Demoras en identificar accesorios y componentes disponibles.

- Solo una persona tenía conocimiento de los accesorios y componentes disponibles.

- Tendencia a realizar pagos fijos sin estimulación del trabajo por el esfuerzo que implicaba determinar salario por resultado.

- Desmotivación del personal por ser más productivos.

- Cierre del período contable una semana después de haberse terminado.

- La contadora se dedica a registrar los asientos contables y menos a realizar proyecciones estratégicas y de gestión.

- No se realizaba control de inventario porque no existía claridad de la cantidad de inventario que debía haber.

- No se puede realizar análisis de rentabilidad de los productos ya que en el momento que eran vendidos no se recordaba el costo de adquisición.

No se registran todas las transacciones del negocio. En este sentido, y basado en el conocimiento de las capacidades de las tecnologías de la información, se han dado cuenta de la necesidad de disponer de un sistema de gestión de información que le permita elevar la eficiencia de su administración con un personal muy reducido.

Ante la necesidad, los actores de este trabajo preparan un proyecto de despliegue del sistema Odoo, donde los directivos estaban dispuestos a restructurar sus procesos de acuerdo al sistema.

Se aplicó la metodología de trabajo expuesta en la sección anterior y finalmente, el proyecto tuvo éxito con mejoras evidentes.

Los procesos de TostoneT pasaron de no tener ninguna aplicación de software a tener un $38 \%$ de sus procesos informatizados para esta primera iteración en el alcance definido.

\section{Informatización}

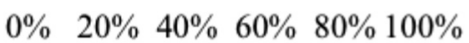

\section{- Informatizado No informatizado}

Figura 12: Grado de informatización.

Fuente: Elaboración propia.
Los cierres contables que antes demoraban más de un mes, actualmente se pueden obtener en tiempo real. Se restructuró el sistema salarial ya que se conoce con mayor claridad la contribución de cada técnico. Creció considerablemente el aprovechamiento de las oportunidades de negocio, ampliando el alcance de los servicios a domicilio, ya que se puede concentrar información específica de los clientes en un solo lugar. El período de duración del proyecto constituyó para sus directivos una etapa de aprendizaje organizacional, ya que durante la implementación fue necesario comprender las buenas prácticas de gestión embebida en el Odoo para su posterior asimilación en el negocio. El nivel de mejora de algunos de los indicadores de gestión y de resultados se muestran en la Tabla 3.

Tabla 3. Indicadores mejorados.

\begin{tabular}{|l|l|}
\hline \multicolumn{1}{|c|}{ Indicadores } & $\begin{array}{c}\text { Nivel de } \\
\text { mejora }\end{array}$ \\
\hline Tiempo de cierre contable & $85,00 \%$ \\
\hline Productividad del trabajo & $23,50 \%$ \\
\hline Tiempo de respuesta a solicitudes de clientes & $81,91 \%$ \\
\hline Promedio de oportunidades gestionadas al mes & $68,00 \%$ \\
\hline Ventas del negocio & $24,00 \%$ \\
\hline Conocimiento de los directivos sobre administración & $36,00 \%$ \\
\hline
\end{tabular}

No solo fueron resueltos los problemas antes mencionados, sino que se incorporaron nuevas capacidades directamente asociada a la planificación de los recursos de la empresa (materiales, información, conocimiento, finanzas, clientes, etc.). (Ver Tabla 4):

Tabla 4. Capacidades incorporadas.

\begin{tabular}{|l|}
\hline Gestión personalizada de los clientes \\
\hline Análisis de ventas y rentabilidad \\
\hline Análisis de productividad y estimulación del resultado \\
\hline Control de los inventarios \\
\hline Estrategia con los inventarios \\
\hline Planificación de compras \\
\hline Gestión de la trazabilidad de productos \\
\hline Gestión de los documentos requeridos por el negocio \\
\hline Gestión de los procesos de negocio \\
\hline
\end{tabular}

\section{CONCLUSIONES}

Existe en Cuba un desarrollo acelerado de MPyMO por su importancia económico y social en el país; 
sin embargo, están presentado problemas de gestión de información que afecta su administración.

Los sistemas ERP crean capacidades de gestión en los pequeños negocios que contribuye a su flexibilidad y agilidad.

La experiencia de trabajo documentada contribuye a la planificación del proyecto de configuración del sistema ERP Odoo en el alcance de la cadena de valor de las pequeñas organizaciones cubanas.

El sistema ERP Odoo configurado en TostoneT, según la experiencia de trabajo documentada, tuvo impactos importantes en su gestión: pues disminuyó el tiempo de cierre contable, aumentó el aprovechamiento de las oportunidades de negocio, así como el conocimiento de sus directivos sobre administración de negocio. Además, aportó nuevas capacidades de gestión y análisis del negocio.

\section{REFERENCIAS}

[1] D.Q. Birkmeier, A. Gehlert, S. Overhage and S. Schlauderer. "Alignment of business and it architectures in the german federal government: A systematic method to identify services from business processes". In System Sciences (HICSS). 46th Hawaii International Conference on System Science, Hawaii, 2013, pp. 3848-3857.

[2] J. García Orozco. "Gestión de la información y el conocimiento: Observatorio para la educación en ambientes virtuales". UDG Virtual. Vol. 134, pp. 53-57. 2010.

[3] E. Yu. "Information systems (in the Internet age)". Practical Handbook of Internet Computing: Boca Raton. 2004.

[4] P.D. Deshmukh, G.T. Thampi and V. R. Kalamkar. "Investigation of quality benefits of ERP implementation in Indian SMEs". Procedia Computer Science. Vol. 49, pp. 220-228. 2015. ISSN: 1877-0509.

[5] Granma. "La informatización de la sociedad, una prioridad para Cuba". In Granma, ed. La Habana, Cuba, pp. 1. 2014.

[6] PCC. "Resolución del VI Congreso del PCC. Lineamientos de la política económica y social del Partido y la Revolución". La Habana. 2011. ISBN: 953-025-152-6.
[7] R.J. Romero Reyes, R. Lugo, S. Dayana and J.B. Velándia. "Impacto de un sistema ERP en la productividad de las PYME". Tecnura. Vol. $16 \mathrm{~N}^{\circ}$ 34, pp. 94-102. 2012. ISSN: 0123-921X.

[8] S. Rus Mata. "Desarrollo de un proceso de selección de un ERP para una PyME de producción y distribución de alimentos". Tesis para optar al grado de doctor. Escuela Técnica Superior de Ingeniería Informática. Universidad Politécnica de Valencia. Valencia, España. 2015.

[9] NC 1030: 2014. "Modelo de gestión para Micro, Pequeña y Medianas Organizaciones". O.N.d. Normalización. 2014.

[10] "Modelo de gestión para micro, pequeñas y medianas organizaciones". 2014.

[11] H.E. Van Auken, B.M. Doran and K.-J. Yoon. "A financial comparison between Korean and US firms: A cross balance sheet canonical correlation analysis". Journal of Small Business Management. Vol. $31 \mathrm{~N}^{\mathrm{o}} 3$, pp. 73. 1993. ISSN: 0047-2778.

[12] S. Vergara. "Pequeñas y medianas empresas en América Latina". Comisión Económica para América Latina y el Caribe, CEPAL, División de Desarrollo Productivo y Empresarial, pp. 21. 2005.

[13] A.F. Antúnez Sánchez, J.M. Martínez Cumbrera and J.L. Ocaña Báez. "Trabajo por Cuenta Propia: incidencias en el nuevo relanzamiento en la aplicación del modelo económico de Cuba en el siglo XXI". Prisma Jurídico. Vol. 14 N$^{\circ}$ 1. 2015. ISSN: 1677-4760.

[14] C. Piñeiro Harnecker. "Nuevas cooperativas cubanas: logros y dificultades". Rebelión. 2015. URL: www.rebelion.org/docs/205163.pdf

[15] L. Rey. Cooperativas no agropecuarias: inconvenientes a enderezar. 2014. 9/3/2015. URL: http://www.trabajadores.cu/20141029/ cooperativas-agropecuarias-inconvenientesenderezar/

[16] MFP. "Resolución No 427/2012”. C. Ministerio de Finanzas y Precios de Cuba, Ed., ed: Gaceta Oficial. 2013.

[17] S.T. Morales-Gualdrón, U.P. Zapata and J.E.D. Urbano. "Factores del servicio y del perfil del emprendedor que inciden en la superviviencia empresarial: una revisión crítica". Revista Lasallista de investigación. Vol. $11 \mathrm{~N}^{\mathrm{o}} 2$, pp. 181-194. 2014. ISSN: 1794-4449. 
[18] A.G. García Cruz. "Levantamiento de procesos y gestión de indicadores para la propuesta de implementación de un sistema ERP en la empresa NOVATECH". Bachelor, Facultad de Ingenierías y Ciencias Agropecuarias, Universidad de las Américas, Quito, Ecuador. 2013.

[19] G. Seo. "Challenges in Implementing Enterprise Resource Planning (ERP) system in Large Organizations: Similarities and differences Between Corporate and University Environment". Composite Information Systems Laboratory (CISL). Sloan School of Management, Massachusetts Institute of Technology Cambridge. 2013.

[20] H.A. Awad. "Maximizing Return on Investment (ROI) of Enterprise Resource Planning Systems (ERP) for Nonprofit Organizations: Proposed Model”. IJCSN International Journal of Computer Science and Network. Vol. $2 \mathrm{~N}^{\mathrm{o}}$ 4. 2013. ISSN: 2277-5420.

[21] M. Porter. "Cadena de valor". México. Editorial CECSA. 2004.

[22] G.N. Purohit, M.P. Jaiswal and M. Pandey. "Challenges involved in implementation of ERP on demand solution: Cloud computing". International Journal of Computer Science Issues. Vol. $9 \mathrm{~N}^{\circ} 4$, pp. 481-489. 2012.
[23] A.X. Ramírez Espinoza. "Análisis comparativo de ERP's con licencia libre, desarrollados en plataforma java e implementación en un caso de estudio para la empresa GRIAND CÍA. LTDA.”. Tesis grado para optar al título de Ingeniero en Sistemas e Informática. Departamento de Ciencias de la computación. Universidad de las Fuerzas Armadas-ESPE. Quito, Ecuador. 2013.

[24] X. Olivan Crego and J.M. Sánchez Castelló. "Estudio para la implantación de un ERP en una PyME". Bachelor, Escola d'Enginyeria. Universitat Autónoma de Barcelona. Barcelona, España. 2015.

[25] C.L. Artola Jarrín. "Estudio de Factibilidad para la Implementación de un ERP como Software como Servicio para la fuerza de ventas de una PYME”. Quito, Ecuador. 2014.

[26] A.E.S. Mateu. "Creación de un portal web con Odoo y un servidor de correo electrónico corporativo para el equipo Formula Student UPV”. Grado en Ingeniería Informática-Grau en Enginyeria Informática Departamento de Organización de Empresas-Departament d'Organització d'Empreses Universitat Politècnica de Valéncia. Escola Técnica Superior d'Enginyeria Informática Valencia, España. 2015.

[27] MFP. "Resolución No 472/2012”. Vol. 003. C. Ministerio de Finanzas y Precios de Cuba, Ed. Gaceta Oficial, pp. 53. 2013. 